\title{
空气质量细粒度数据内联关系可视分析系统
}

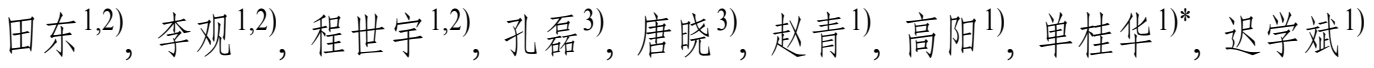 \\ 1) (中国科学院计算机网络信息中心先进交互式技术与应用实验室 北京 100190) \\ 2) (中国科学院大学 北京 100049) \\ 3) (中国科学院大气物理研究所大气边界层物理和大气化学国家重点实验室 北京 100029) \\ (sgh@cnic.cn)
}

\begin{abstract}
摘 要: 随着数据观测和数字计算技术的快速发展, 空气质量数据粒度越来越细致, 为研究空气质量的细粒度数据 内联关系可视化、辅助理解空气污染物的转化规律提供了数据基础. 针对蕴含丰富内联特征的大气质量细粒度数据 开发了一个内联关系可视分析系统. 首先, 基于大气质量数据多维属性设计时空数据维度模型增强数据的时空表达, 并提出一种基于深度学习的特征提取方法，将空气质量数据从高维空间映射到支持交互可视分析的低维特征空间; 然后, 设计了一套多视图联动的可视化系统, 帮助用户发现空气污染传输中的细粒度内联关系, 理解污染传输途径 的数据分布特征. 用户实例和用户评价结果表明, 该系统是有效的.
\end{abstract}

关键词：空气污染; 可视分析系统; 轨迹可视化; 比较可视化

中图法分类号: TP391.41 DOI: 10.3724/SP.J.1089.2021.18979

\section{Visual Analysis System for Fine-Grained Inline Relationship of Air Quality Data}

\author{
Tian Dong ${ }^{1,2)}$, Li Guan ${ }^{1,2)}$, Cheng Shiyu ${ }^{1,2)}$, Kong Lei $^{3)}$, Tang Xiao ${ }^{3)}$, Zhao Qing ${ }^{1)}$, Gao Yang ${ }^{1)}$, Shan Guihua ${ }^{1 *}$, \\ and Chi Xuebin ${ }^{1)}$

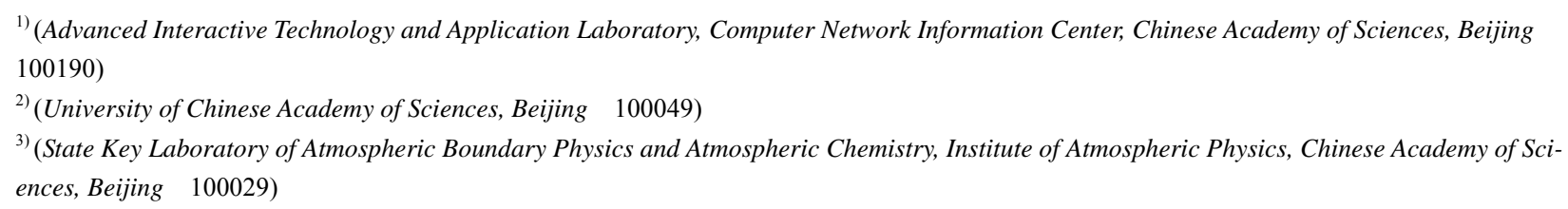

\begin{abstract}
With the rapid development of data observation and digital computing technology, the granularity of air quality data tends to be fine, which provides a data basis for visualizing data of air quality and understanding the transformation law of air pollutants. This paper proposes a visual analysis system for inline features of fine-grained air quality data. We design a spatial-temporal data dimensional model to enhance the spatial-temporal expression of the data. We propose a deep learning based scheme that embeds high-dimensional air quality data into a low-dimensional feature space, which supports interactive visual analysis. We implement a multi-view linkage visualization system which assists discover the fine-grained inline relationship in the spread of air pollution and understand the data distribution characteristics of pollution transmission routes.
\end{abstract}

收稿日期: 2021-03-08; 修回日期: 2021-03-15. 基金项目: 国家重点研发计划(2016YFB0200804); 中国科学院战略性先导科技 专项(A 类)(XDA19080102); 中国科学院青年创新促进会(2018203). 田东(1983-), 男, 硕士, 高级工程师, 硕士生导师, CCF 会员, 主要研究方向为大数据可视化与可视分析; 李观(1990-), 男, 博士研究生, 主要研究方向为大数据可视化与可视分析; 程世宇 (1993-), 男, 博士研究生, 主要研究方向为大数据可视化与可视分析; 孔否(1994-), 男, 博士研究生, 主要研究方向为大气化学资 料同化; 唐晓(1981一), 男, 博士, 副研究员, 硕士生导师, 主要研究方向为大气化学资料同化和集合预报; 赵青(1984一), 女, 硕士, 工程师, 主要研究方向为数据可视化; 高阳(1987-), 女, 硕士, 工程师, 主要研究方向为数据可视化; 单桂华(1976一), 女, 博士, 研 究员, 硕士生导师, CCF 会员, 论文通讯作者, 主要研究方向为大数据可视化与可视分析; 迟学斌(1963一), 男, 博士, 研究员, 博士 生导师, CCF 杰出会员, 主要研究方向为高性能计算与网格技术. 
User cases and user evaluation results show that our system is effective.

Key words: air pollution; visual analysis system; trajectory visualization; comparative visualization

大多数发展中国家都面临着空气污染问题, 严重影响着人们的健康 ${ }^{[1-2]}$. 我国面临的大气复合 污染问题非常复杂，涉及气象、排放、化学、大气 传输和污染管控等多种复杂因素的综合作用，非 线性强, 理清其内联关系是一大挑战, 也是大气污 染治理的关键. 将内联关系分析与可视化结合，有 助于科研人员和管理人员直观理解这一重要信息. 现有大气观测数据的粒度不足, 主要是由于观测 点分布离散, 不能充分考虑地形等细粒度因素的 影响. 数据粒度粗糙, 导致环境治理能力不足 ${ }^{[3-4]}$. 现有内联分析方法的不足是由于空气污染普遍存 在明显的传输扩散特性 ${ }^{[5]}$, 并且扩散过程中存在多 维因素之间的相互影响，导致传输轨迹的不确定 性. 上述问题对大气污染的共同预防和控制具有 重要意义 ${ }^{[6]}$.

目前, 基于细粒度数据的空气污染研究仍存 在诸多挑战. (1) 探索空气污染区域的细粒度分布 需要更加精准的特征提取方法. 特征提取方法为 污染分布的精确划分和传输轨迹的发现提供帮助, 且为防治计划的制定提供技术参考. (2) 分析空气 污染轨迹上的属性关系, 需要领域专家的经验指 导. 为了让领域专家较好地利用经验分析, 需要设 计一套针对细粒度空气质量数据的可视分析系统. 领域科学家需要通过简洁的参数设计和交互, 便 捷查看每种大气元素, 如二氧化氮 $\left(\mathrm{NO}_{2}\right)$, 臭氧 $\left(\mathrm{O}_{3}\right)$ 和 $\mathrm{PM}_{2.5}$ 的地域分布. 同时，系统需支持交互 式地对大气全要素数据进行内联特征提取、聚类分 析和比较分析. 通过多视图联动的方式实现对高 维大气数据的探索式分析, 帮助领域专家发现重污 染区域的空间分布特征，分析特定传输轨迹上各大 气要素的影响程度.

针对第 1 个挑战，本文设计了一种针对细粒度 空气质量数据的内联特征提取方法. 基于深度学 习方法, 得到高维空气质量数据的内联特征, 该内 联特征可以较好地区分不同的空气污染区域. 针 对第 2 个挑战, 本文设计了一个雾霾质量可视化分 析系统 (haze quality visualization system, HAQVIS), 帮助研究人员对细粒度的时空多维空气质量数据 进行探索式可视分析. 本文的主要研究内容如下.

(1) 基于变分自编码器(variational auto-encoder,
VAE)的空气质量数据特征提取方法, 可用于获取 内联特征，实现空气污染分布的精确划分.

(2) 针对细粒度空气质量数据的可视分析系 统, 可以探索性地分析高维空气质量数据的精细 内联关系

\section{1 相关工作}

多维数据可视化技术中特征提取方法的基本 思想是将高维空间中的数据映射为低维空间中的 点, 且维度之间的某种关系保持不变. 因此, 关系 定义不同，特征提取方法也不同 ${ }^{[7]}$.

可视化方法是多维数据分析的有效方法. $\mathrm{Qu}$ 等 ${ }^{[8]}$ 开发了一套可视化分析系统，通过特别设计的 圆形条形图和加权图分析香港的空气质量. Trein$\mathrm{ish}^{[9]}$ 设计了一种大气流场可视化方法, 以分析关 键点和基于图像的种子点位置. Völgyesi 等 ${ }^{[10]}$ 设计 了基于车辆传感器数据的空气质量图可视化分析 系统, 以快速获得空气质量的可视化图像. 此外, 可视化数据融合的方法被用来解决可视化设计问 题 ${ }^{[11]}$, 层次线性模型用于获得适用于层次分析法的 多层结构. Healey 等 ${ }^{[12]}$ 提出了一种使用画笔笔画对 多维天气数据进行可视化分析的方法. Ito 等 ${ }^{[13]}$ 利用 箱形图和时间间隔相关图表示气象监测站的大气 数据. Tang 等 ${ }^{[14]}$ 用可控的纹理合成技术对大气数 据进行可视化编码. Demuzere 等 ${ }^{[15]}$ 设计了一种空 气质量预测工具，通过地图和等高线等可视化方 法分析 $\mathrm{O}_{3}$ 和 $\mathrm{PM}_{10}$ 对大气和天气的影响. 编码技术 也常被用于高维数据可视化, Quinan 等 ${ }^{[16]}$ 提出了 一系列编码方法解释各种天气特征及其相互作用, 并以直观的方式进行天气预报。然而，这些通用方 法在确保聚类结果的准确性方面存在一些缺陷, 如缺乏具有高可靠性的非线性降维方法、缺少传输 轨迹的属性趋势和相关对比方法, 以及缺乏具有 斜率选择的多元相关分析(非线性)方法等. 而这些 是大气领域专家进行大气数据关联分析的迫切需 求. 本文提出了一个基于 VAE 的内联关系特征提 取模型，并设计了可用于交互分析的多个图形，以 实现上述功能分析. 


\section{2 内联特征提取}

使用中国科学院发布的空气质量再分析数据, 包括每个网格点上的空气成分属性、风场、温度、 压力场和其他环境数据, 以及经度、纬度和海拔等 空间数据 ${ }^{[17]}$. 针对数据特点, 设计实现了一个数 据处理流程, 以支持可视化系统的数据展示.

空气质量细粒度数据内联可视分析系统的流 程如图 1 所示, 将输人的空气质量再分析数据进行 时空维度数据计算和数据添加, 并将得到的多维 数据通过 VAE 模型进行特征提取处理和聚类, 以 分析数据的内部联系. 用户界面(user interface, UI) 交互与可视化模块对提取的特征数据和聚类结果 进行关联分析.

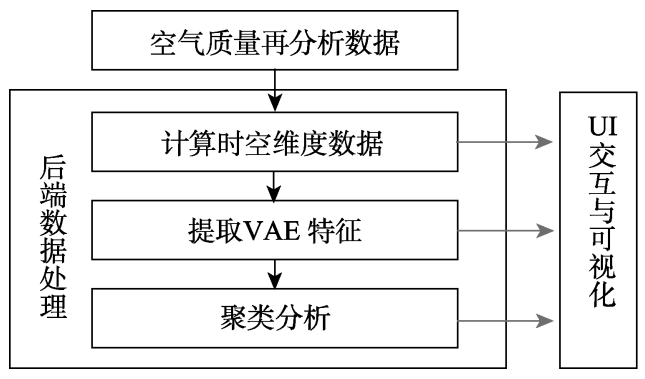

图 1 本文系统流程

\section{1 时空维度计算模块}

由于已有的空气质量再分析数据缺少时空分 布特征，因此在原有数据基础上，增加时间维度和 空间维度信息, 分别用来描述当前时间的变化程 度和当前点的空间变化程度。

\subsection{1 时间维度数据计算}

时间(TIME)维度用来增强数据对时间变化特 征的表达. 空气污染程度主要由 $\mathrm{PM}_{2.5}$ 数据反映, 污染物随时间的传输是研究人员的关注点. 其中, $\mathrm{PM}_{2.5}$ 值 0 35 $\mu \mathrm{g}$ 代表空气质量非常好; 35 75 $\mu \mathrm{g}$ 为 良好; 75 115 $\mu \mathrm{g}$ 为轻度污染; 115 150 $\mu \mathrm{g}$ 为中度污 染; $150 \sim 250 \mu \mathrm{g}$ 为重度污染; 超过 $250 \mu \mathrm{g}$ 为严重污 染. 不同地区 $\mathrm{PM}_{2.5}$ 值的变化反映了该地区的污染 程度. 了解不同区域的变化趋势可以帮助分析不 同的污染变化模式，其计算公式为

$$
f^{\prime}\left(t_{i}\right)=\frac{-f\left(t_{i+2}\right)+8 f\left(t_{i+1}\right)-8 f\left(t_{i-1}\right)+f\left(t_{i-2}\right)}{12 h} .
$$

其中, $h$ 表示一个时间刻度; $f(t)$ 表示 $t$ 时刻污染物 浓度值. 根据不同的时间粒度(小时、日、月、年)，选 取当前时间前后 4 个时刻的值, 计算出每个网格点 上 $\mathrm{PM}_{2.5}$ 值的变化趋势，得到当前点的时间属性.

\subsection{2 空间维度数据计算}

增加空间 (SPACE)维度的主要目的是增强数 据对空间变化特征的表达能力. 地形的高度会影 响垂直方向的空气特性, 如高原地区的污染通常 很小. 但是, 高度不能反映周围地形的水平波动特 征. 地形起伏代表该区域的宏观地形特征，基于地 形起伏的概念, 设计了一个新的属性表示当前网 格点周围地形的起伏, 即区域中最高点的高度 $H_{\text {max }}$ 和最低点的高度 $H_{\text {min }}$ 的差值, 其计算公式为 $S=H_{\text {max }}-H_{\text {min }}$. 通过提取当前点的 8 个邻域点的 高度值, 计算它们的最大值和最小值并求差, 以得 出地形起伏值 $S$. 遍历所有网格点以获得新的数 据维度信息(SPACE). 该变量能够表示地形波动对 大气质量的影响.

图 2 所示为新增的 2 个维度数据在某一时间的 热力图效果.

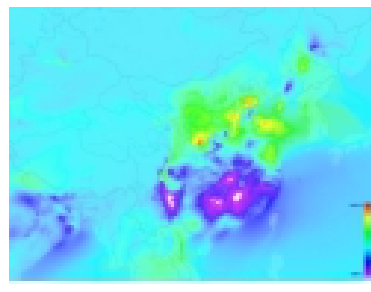

a. TIME

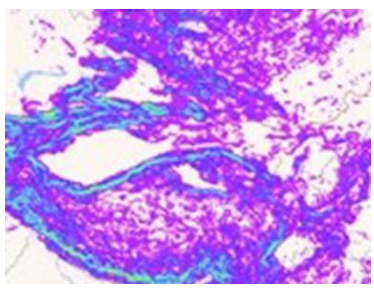

b. SPACE
图 2 时空数据热力图

\section{2 内联特征提取流程}

使用基于深度学习模型的 VAE 作为数据特征 提取方法, 能够针对规格大小不同的数据进行内 联特征提取.

\section{2 .1 输人类型}

输人数据类型由空气质量数据和时空维度数 据组成. 时空维度计算模块处理后得到 16 维输人 数据, 如图 3 所示. 图 3a 图 3p 分别为温度(TEMP), $\mathrm{PM}_{2.5}$ 、二氧化硫 $\left(\mathrm{SO}_{2}\right), \mathrm{O}_{3}, \mathrm{NO}_{2}$ 、一氧化碳 $(\mathrm{CO})$ 、 水平风速 $(U)$ 、垂直风速 $(V)$ 、经度 $(L O N)$ 、纬度 $(\mathrm{LAT})$ 、海拔高度(HEIGHT), $\mathrm{PM}_{10}$ 、湿度 $(\mathrm{RH}) 、$ 压 强(PSFC), TIME, SPACE. 数据网格中的每个网格 点都有 16 个值, 对应于一组数据, 反映了当前区 域的大气质量, 它们之间存在线性或非线性关系. 在考虑了主成分分析法(principal component analysis，PCA)， $t$-分布领域嵌人方法( $t$-distributed stochastic neighbor embedding, $t$-SNE)和其他数据降 维方法之后, 选择了基于 VAE 的方法提取数据的 特征. 在编码过程中, VAE 既能表征线性变换, 也 能表征非线性变换; 而 PCA 只能执行线性变换. 
通过设置合适的维度和稀疏约束, VAE 可以学习到 比 PCA 和 $t$-SNE 等技术更有意义的数据投影. 由 于 VAE 同时考虑了线性和非线性因素，因此可以 验证特征数据是否通过编码器和解码器反映了原 始数据的特征. 这是其他方法无法达到的效果, 也 为大气数据的特征可追溯性和可解释性提供了技 术支撑。

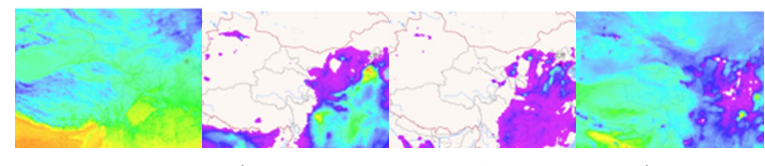

a. TEMP

b. $\mathrm{PM}_{2.5}$

c. $\mathrm{SO}_{2}$

d. $\mathrm{O}_{3}$

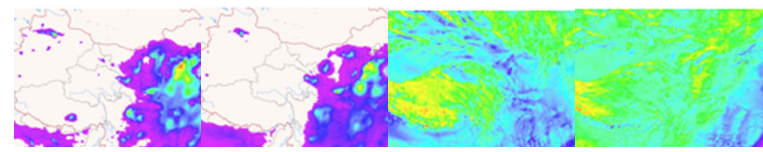

e. $\mathrm{NO}_{2}$

f. $\mathrm{CO}$

g. U

h. V

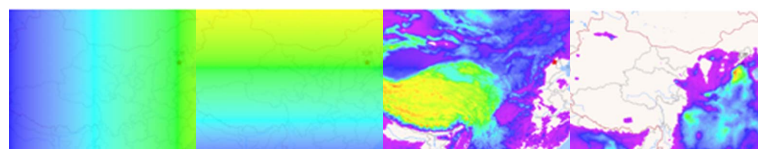

i. $\mathrm{LON}$

j. LAT

k. HEIGHT

1. $\mathrm{PM}_{10}$

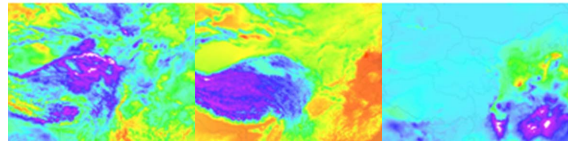

m. RH

o. TIME
图 316 个维度的输人数据模型

数据的维数不同, 数据的取值范围和分布有 也较大差异. 选择 $Z$ 分数归一化方法处理数据, 并 获得特征提取模型的标准输人数据.

\subsection{2 特征提取模块}

特征提取方法的主要原理如下，内联特征提 取模块的输人是 16 维数组, 而输出是低维坐标数 组. 在编码器部分, 使用全连接层将 16 个维度编 码为 8 个维度, 然后通过线性整流函数(rectified linear unit, ReLU)激活功能层处理, 使用全连接层 处理, 进一步将 8 维编码为 2 个维度. 在解码器部 分, 隐藏变量的 2 维空间坐标通过全连接层转换为 16 维, 最后输出最终结果.

该模块中使用交叉熵方法计算重建损失，总 损失是重建损失与 KL 散度之和. 深度学习网络会 进行多次迭代，直到损失函数趋于平缓为止. 特征 提取方法步骤如图 4 所示.

使用训练后的网络重构整个网格点 $O$, 获得 重构值 $C$, 并输出隐空间中每个网格点的坐标集 $Z$. 其中, $O$ 和 $C$ 用于分析和验证特征提取效果, $Z$ 用于进行数据的交互式可视化分析 ${ }^{[18]}$.

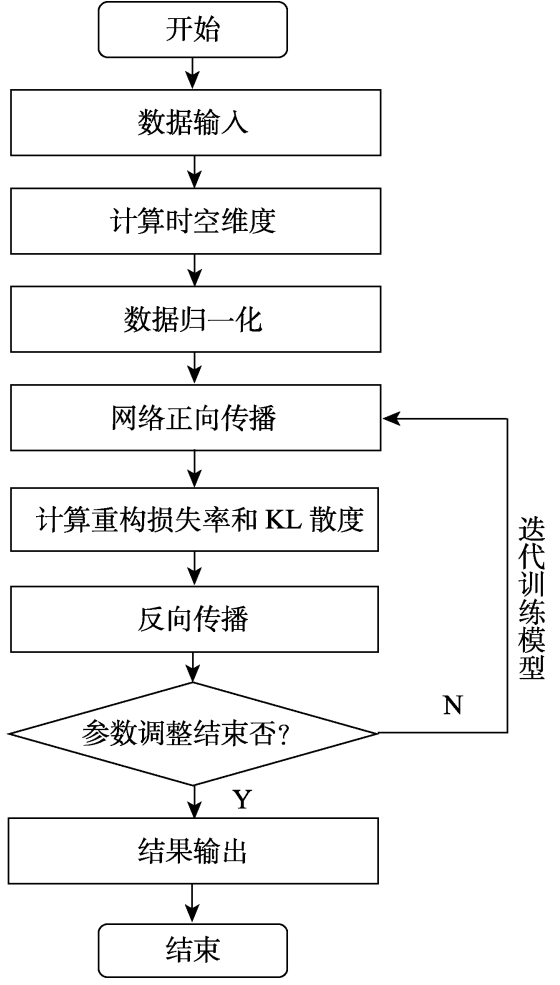

图 4 特征提取方法流程

\subsection{3 数据聚类模块}

特征提取得到的隐含变量, 需要进行可视化 交互. 为了给可视化交互提供参考和引导辅助, 使 用聚类方法对隐变量进行划分. 数据的特征提取 和聚类方法的结合可以帮助研究人员快速获得数 据的分布特征，同时指导用户对类内和类间的数 据进行交互式分析. 为了实现聚类可视分析功能, 选择 3 种不同特点的聚类方法, 其中, Birch 方法可 以有效地识别噪声点并过滤; $K$-means 聚类具有聚 类效果好、解释能力强的优点; 高斯混合聚类无法 获得确定的分类，但是可以得到每种分类的概率. 聚类方法为可视化交互式分析提供了更多的交叉 参考.

\section{3 可视化设计}

为了解决领域专家对数据分析的需求，基于 上述数据处理流程、特征提取模块和聚类模块, 设 计了 HAQVIS，如图 5 所示. 数据包括第 2.1 节中 提到的原始数据、提取的特征数据和聚类后的数 据. 领域专家从系统设计和实现的初始阶段开始, 全程参与了该工作的需求设计并提供了指导

如图 5 所示, 该系统包括 5 个部分, 其中(1)表 示参数选择器, 用于整个系统的可选参数设置; 


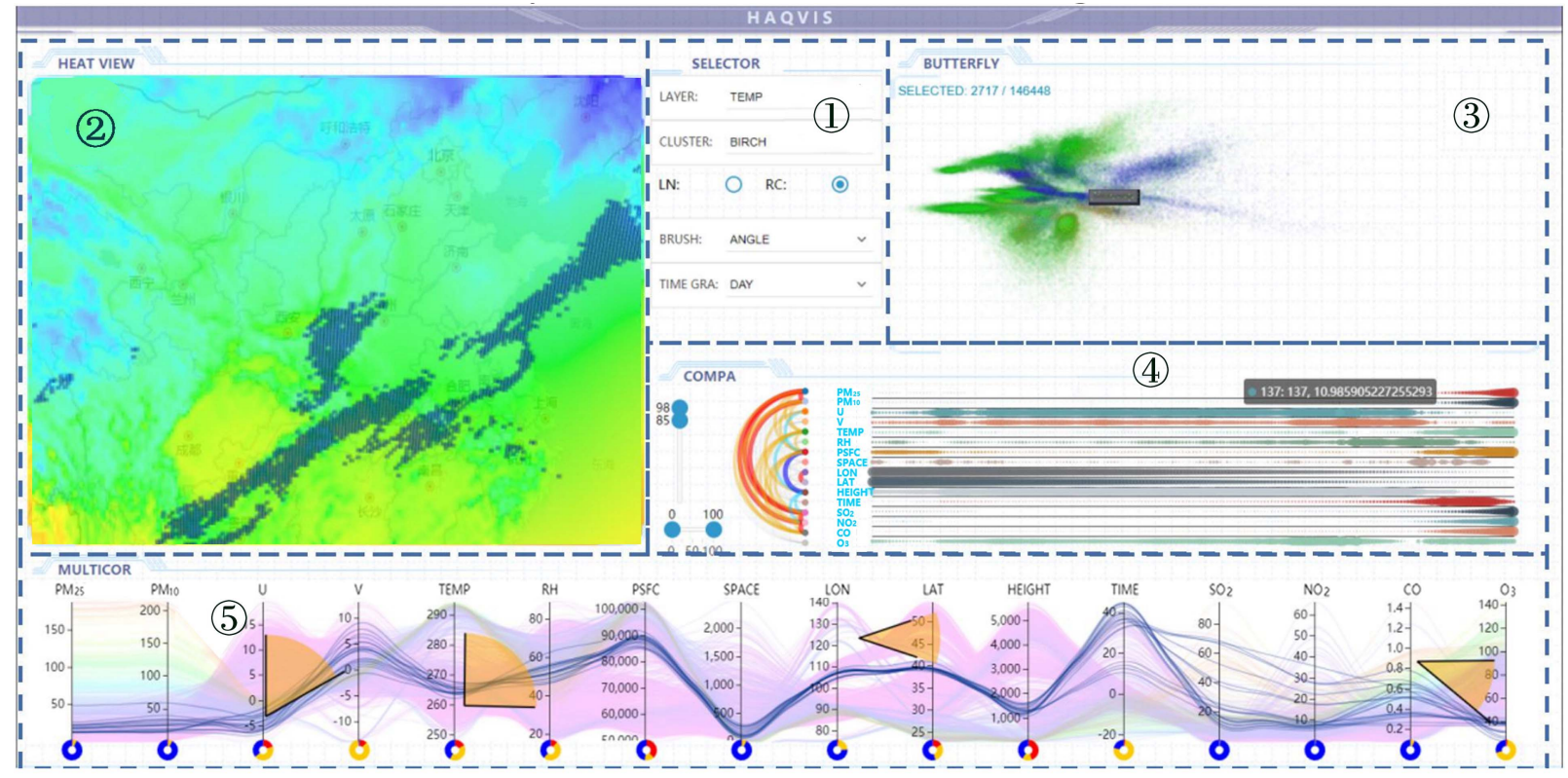

图 5 HAQVIS 系统界面

(2)表示热图视图，用于数据的可视化和交互; (3) 表示蝶形视图, 用于特征提取数据的可视化和交 互; (4)表示比较视图, 用于数据序列的比较和相关 性分析; (5)表示多维联动视图, 用于多维关联数据 的可视化.

\section{1 参数选择器}

面板中有 5 个选择器, 它们用来调整视图中图 形的各项参数，设置可视化着色方式和值域过滤. LAYER 项在热图视图中实现对不同数据层的选 择，下拉列表包括 16 个维度的名称供选择. CLUSTER 项在特征视图中实现了聚类方法的选 择，包括第 2.2.3 节介绍的 3 种聚类方法，默认使用 Birch 方法聚类. 第 3 个选择器用于热图视图交互 模式选择，LN 代表图形上的绘制轨迹，轨迹上的 返回点; RC 代表图形上的绘制矩形，矩形区域中 的返回点. BRUSH 项在多维联动视图中实现平行 坐标的笔刷类型选择，包括一维单选、一维多选、 线段选择和角度选择. TIME GRA 项目实现时间粒 度的选择. 不同的时间粒度表示数据时间的采样 粒度，默认为天.

\section{2 热图视图}

热图视图用来详细显示每项空气质量维度数 据, 反映单个维度在地理空间上的分布状态. 如图 6 所示, 通过颜色映射可视化当前选定维度的数 据. 同时, 聚类区域的亮点可以实现对特定分类数 据的空间可视化分析.

基于热图视图进行开发和设计，基础地图采 用百度地图和卫星地图，可以通过右上角的单选

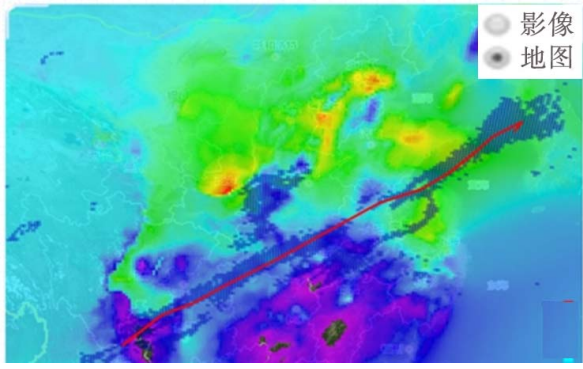

图 6 热图视图可视化

按钮进行切换. 地图支持使用基础地图进行缩放 和转换，该地图可以查看国家/地区的总体分布， 也可以向下钻取到特定区域以查看数据的可视化 细节. 在颜色表设计方面, 通过对 16 维数据的分 析, 确定了可以很好地区分所有维度的彩虹颜色 表. 同时，对大比例、小数值的数据采用动态透明 设计. 当该值小于某个阈值时，随着该值的减小， 颜色逐渐变得更透明，因此最小值在热图中完全 透明. 这种设计不仅确保了主要数据特征的可视 化, 而且还尽可能地公开了底图和地理标志信息, 以便更好地观察数据的空间分布.

本文设计了点选择和线选择 2 种交互模式，方 便用户选择交互数据. 当鼠标在地图上滑动时, 将 实时返回与当前鼠标点相对应的网格信息. 线选 择方法是通过鼠标点选以返回轨迹上所有点的网 格信息，从而选择某条具体轨迹.

对于其他视图选择数据, 设计了一种网格突 出显示方法, 所选区域覆盖有规则的格子纹理, 以 避免由于热图的影响而难以区分所选区域. 


\section{3 特征视图}

特征视图提供对大量的空气质量特征提取数 据进行可视化和交互. 由于对于测试的数据分布 大多具有类似蝴蝶的形状，所以又称为蝶形视图， 该视图旨在提取可视化特征数据和聚类数据, 并 进行交互，如图 7 所示. 设计颜色阵列表示不同的 分类，从而可以直观地区分聚类. 图形的绘制是由 WebGL 开发的, 它保证了在浏览器中呈现成千上 万个数据点的能力, 并支持实时、快速地绘制大量 的点数据.

在视图中绘制特征提取数据后，在数据密集 区域，大量数据点落在相同的像素点上. 为了避免 交互时发生遮挡，使用了一个自适应透明颜色表.

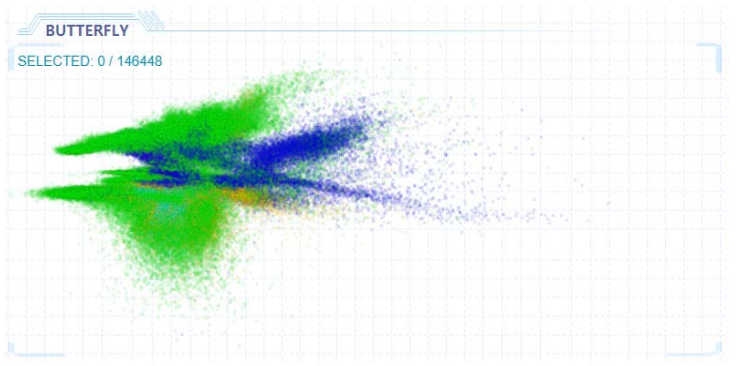

图 7 特征视图
如果未选择任何区域, 则使用较低的透明度更好 地呈现数据整体分布; 选择区域后，切换较高的透 明度以突出显示所选区域. 这样, 在突出显示所选 区域的位置的同时, 可以更直观地查看数据的聚 类，为下一步的交互式选择提供参考.

视图支持点选择和框选择 2 种交互模式. 为了 快速选择鼠标悬停状态下的点, 并避免频繁遍历 所有点, 设计了一种点序列缓存方法. 首先, 初始 化与当前视图像素的长度和宽度一致的数组. 绘 制图形时, 在数组中记录落在像素点上的数据的 序列号. 当鼠标悬停时, 用户可以快速地读取存储 在数组中的序列号, 以实现快速检索. 在框选择模 式下，仍然使用数据遍历的方法，因此此时选择的 数据更加准确, 即使存在遮挡, 也可以选择. 选择 后，在左上角显示所选点数的比例.

\section{4 比较视图}

比较视图用来对重要的空气质量维度数据进 行对比, 如图 8 所示. 通过此视图, 系统可以比较 序列数据的多个维度, 同时可以调整维度的相关 性分析. 在图的右侧, 气泡图用于可视化数据系列 的 16 维数据. 为了使气泡的最大半径保持一致, 使用 min-max 标准化方法对展示数据进行标准化.

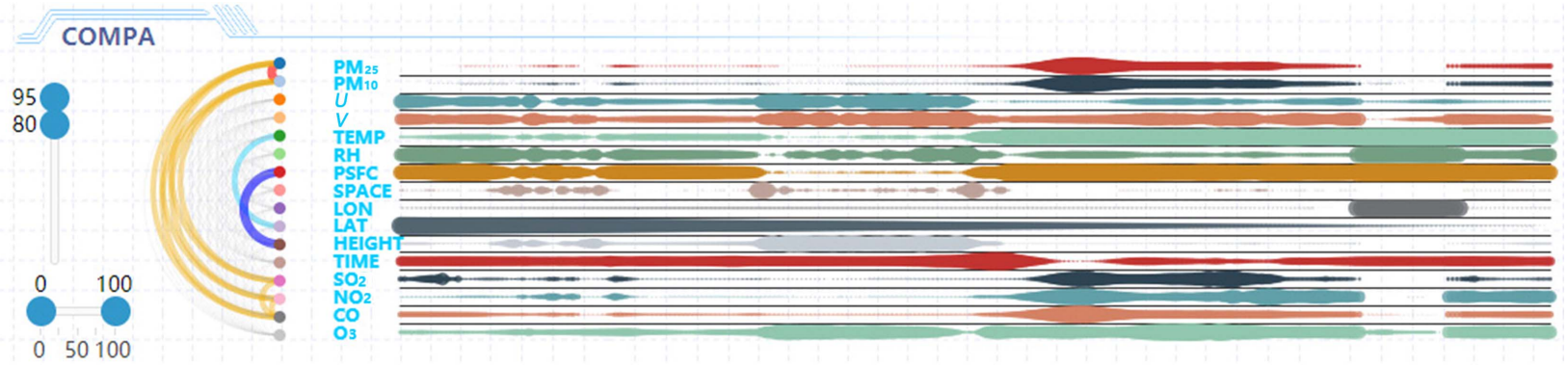

图 8 用于数据对比的比较视图

在空气质量数据分析中, 不同领域的数据之 间是否存在线性关系对于领域专家而言非常重要. 本文基于 Pearson 相关系数方法设计了一个可视化 视图, 如图 8 左侧所示. 视图的中间部分是 16 个维 度的名称，它对应于右侧维度的数据变化趋势图. 左侧为关联视图. 每 2 个维度通过半圆连接. 半圆 的颜色和粗细代表 2 个维度间的相关系数. 本文的 相关绝对值定义了 2 个國值: 数值大的用 PCC_high 表示, 数值小的用 PCC low 表示. 如果其数值大 于 PCC high, 则连接最粗的线; 如果其数值在 PCC_high 和 PCC_low 之间, 则用细线连接; 如果 其数值小于 PCC low, 则隐藏连接线. 同样, 将红
色和黄色用于正相关, 将蓝色和紫色用于负相关.

考虑研究人员需要选择性观察不同的相关性, 系统提供了國值双轴选择模块. 图 8 的左侧有 2 个 滑块：下面的滑块用于设置可以显示的相关系数 区域，此区域外的连接线将被隐藏; 上面的滑块用 于设置 2 个國值 PCC_high 和 PCC_low.

\section{5 多维联动视图}

多维联动视图用于所有相关数据维度的定性 聚类和关联分析. 在平行坐标的基础上添加了一 种新的可视化方法. 数据在每个轴上的分布对于 数据分析非常重要, 但是当数据量很大时, 直接在 坐标轴上绘制直方图的方式很容易产生遮挡, 并 
且大多数情况下用户只关注数据的粗略分布. 本 文设计了一种可视化的方法, 即在每个轴的底部 添加一个饼图, 将每个数据轴分为 3 个部分, 对 3 个相等部分上的数据分布进行计数, 并将这 3 个值 映射到相应的饼图. 如图 9 所示, 从高到低的颜色 分别是红色、绿色和蓝色，上述设计可帮助用户快 速地了解每个轴上当前数据的分布.

平行坐标渲染采用渐变渲染的方式, 无论数 据量有多大, 它都可以确保图像在渲染过程中可 以随时与图像进行交互. 同时, 设计了 4 种画笔选 择方法: 1 维单选、 1 维多选、笔刷选择和角度选择; 其中, 角度选择根据相邻 2 维数据的变化剧烈程度 对数据进行过滤.

\section{6 协同交互设计}

交互设计保证了各个视图之间的数据联动.
本文设计了一个统一的数据接口, 以实现热图视 图、蝶形视图、多维联动视图和比较视图 4 种视图 之间的数据交互和协同分析, 热图视图、蝶形视图 和多维联动视图均以网格的索引值作为输人. 选 择视图后, 所选数据的索引数据将更新并应用于 其他视图, 以完成数据链接. 在比较视图接管后, 从热图视图传人的轨迹索引值将可视化轨迹上的 点阵列.

为了保证系统运行效率，特征视图利用渐进 式渲染的方式实现了多个视图之间的联动. 多维 联动视图支持不阻塞的渲染方式，即选择某个区 域后, 线元素持续添加到可视化视图中来, 不阻塞 主线程，更不会导致程序等待超时，既保证了十万 多个点的实时渲染, 也保证了大量点的实时交互 选择.

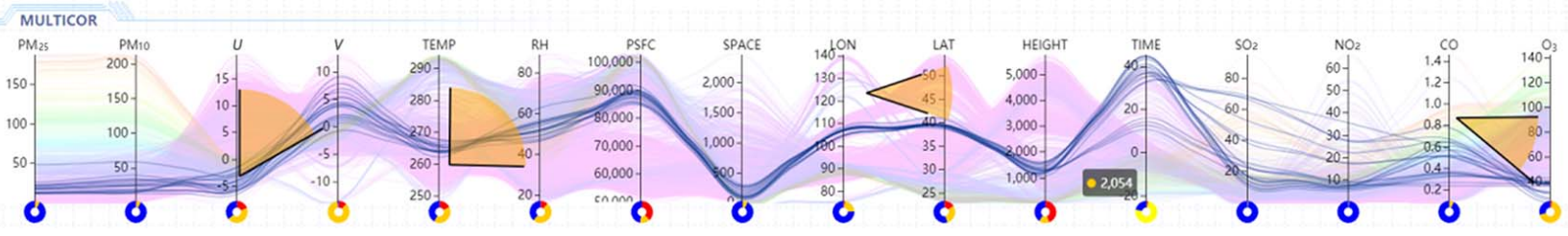

图 9 多维联动视图

\section{4 实 例}

大气领域的相关研究人员使用该系统对空气 质量数据进行可视化分析, 其应用范围包括污染 区域的空间划分、组成轨迹分析和多元关联分析; 其中，网格大小为 $339 \times 432$, 共有 146448 个数据点.

下面从污染空间划分、传输轨迹分析和多要素 关联分析 3 个实例，进而阐述本系统对细粒度空气 质量数据进行内联关系可视分析的方法.

\section{1 污染空间划分}

污染空间划分反映了细粒度空气质量数据的 污染指标和各维度数据的内联关系. 精确的区域 聚类方法可以探索导致重污染的各大气要素间细 粒度的内联关系分布，研究区域划分对于研究人 员深人研究污染的深层原因和防止雾䨪具有重要 的参考价值.

通过热图视图和蝶形视图的联动，可视化系 统可以帮助用户实现区域聚类分析，在隐变量空 间中进行交互式选择聚类，并且可以在空间视图 中突出显示当前选择的区域，从而可以直观地对 不同污染情况下空气质量分析，尤其是污染空间 的分区分析

\subsection{1 重污染区划分}

在蝶形视图中可查看多个数据聚合区域. 如 图 10 所示, 在参数设置面板中选择 RECT 选项, 打开矩形选择工具. 当通过蝶形视图中的 RECT 选择工具选择蝴蝶的腹部时, 可以在热图视图中 观察选定区域中晶格数据的空间分布. 此时, 将 热图视图的基本图切换为 $\mathrm{PM}_{2.5}$ 数据的热图. 从 图中可以发现, 蝴蝶腹部区域的点很好地覆盖了 我国东北 $(A)$ 、京津冀 $(B)$ 、四川盆地 $(C)$ 、南部腹 地 $(\mathrm{E})$ 、乌鲁木齐 $(\mathrm{F})$, 以及印度加尔各答 $(\mathrm{D})$ 等雾 需严重的地区, 其中图中 SELECTED 标注的数字 表示当前选中的数据点占总数据点的比例. 此外, 4 个区域 A， B , C , D 之间具有明显的内联关系, 导致类似于传输通道的明显分布, 这对于大气研 究者划分该分布区域具有重要的参考意义. 仅区

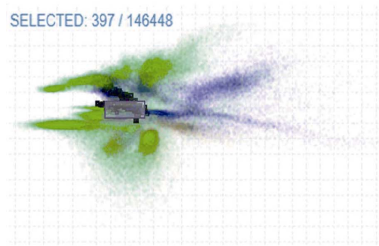

a. 选择区域

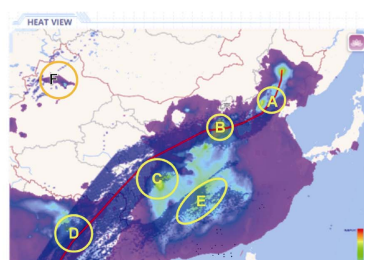

b. 图 $\mathrm{a}$ 的热图分布状态
图 10 污染区在特征视图上的空间分布 
域 $\mathrm{C}$ 的位置略有偏离, 该区域以西是青藏高原地 区，以东是四川盆地; 同时, 该区域的大规模地形 波动并未反映在模型输人数据中, 这为研究高原 和盆地对污染输送的影响提供了重要线索.

聚类显示，乌鲁木齐的区域 $\mathrm{F}$ 是西部地区污染 程度较高的区域.上述不同区域的污染因子是不同 的，但通过可视化分析发现，这些严重污染的区域 在空气质量的隐含特征空间中具有高度相似性.

\subsection{2 污染空间异常发现}

通过矩形选择工具在蝶形视图中选择蝴蝶的 下翼，在热图视图中看到聚类区域的空间分布，如 图 11 所示. 可以发现该区域由 2 部分组成，且位于 内陆和海洋相距较远的地方, 一个在中国的西部 (A), 另一个在东边的太平洋上(B), 空间距离差 异很大. 但是通过聚类分析，在特征空间中将 2 个区域分为一组, 表明 2 个区域在空气质量上具 有很强的相似性，这对空气污染研究具有重要的 参考意义。

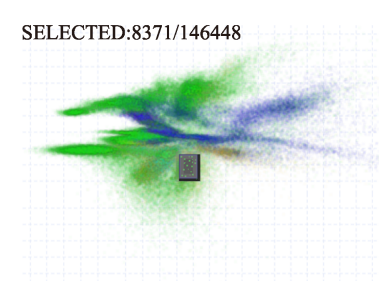

a. 选择蝶形视图下翼

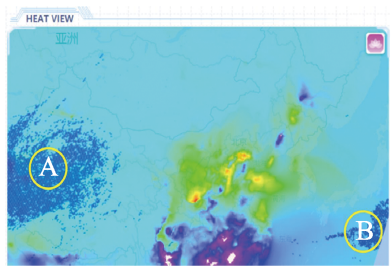

b. 团簇分布
图 11 发现异常区域

\section{2 传输轨迹分析}

传输轨迹分析探索细粒度空气质量数据在感 兴趣传输轨迹各维度之间的内联关系. 定向污染 转移轨迹属性关系通过交互式的轨迹选择和轨迹 上空气质量分布的比较分析方法进行挖掘, 为研 究人员研究污染传输轨迹的空间趋势、发现污染物 之间的相关性、制订有针对性的防治计划提供技术 手段. $\mathrm{PM}_{2.5}$ 的运输轨迹分析对于防止污染非常重 要，污染物在特定轨迹上的分布以及轨迹上不同
维度的数据是否具有线性相关性是研究人员不断 解决的问题.

首先，选择 $\mathrm{PM}_{2.5}$ 作为数据维度绘制热图, 然 后使用轨迹绘制工具在热图视图中绘制从污染中 心到无污染区域的轨迹. 在视图中，用户可以看到 隐变量空间中当前轨迹上数据点的趋势. 除了分 布在蝴蝶的腹部区域外, 它还扩展到蝴蝶的头部 区域，如图 12 所示.

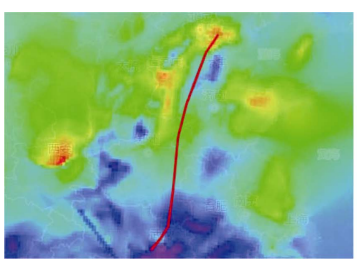

a. 传输轨迹选择

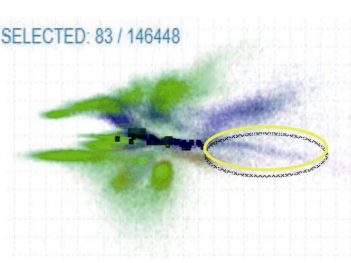

b. 轨迹点位于蝴蝶腹部的中线
图 12 传输轨迹图和群集

同时，在比较视图中，可以沿着轨迹上的采样 点查看每个元素的分布趋势, 如图 13 所示. 在图 13 的右侧可以看到, 在该传输轨迹中, 海拔高度 从低到高分布, 轨迹起点处的 $\mathrm{SO}_{2}$ 和 $\mathrm{NO}_{2}$ 较高, 而 轨迹中间的 $\mathrm{O}_{3}$ 浓度较高, 温度最低. 在图 13 的左 侧, 每 2 个元素之间存在定量线性相关性. $\mathrm{SO}_{2}$ 与 海拔高度呈极显著的负相关, 即海拔越高, $\mathrm{SO}_{2}$ 含 量越低. 高度与 $\mathrm{PM}_{2.5}$ 和 $\mathrm{PM}_{10}$ 也显示出很强的负相 关性; $\mathrm{SO}_{2}$ 和 $\mathrm{PM}_{2.5}$ 之间有一条粗线, 表明它们之间 有很强的正相关性. 通过调整左侧的滤波器参数, 可以调整相关性显示范围, 以更好地观察相关性.

系统还可以使用多维联动视图观察轨迹数据 元素之间的相关性. 如图 14 所示, 在此轨迹上, 海 拔高度对污染程度有显著影响, 污染越严重, 趋势 越差; 重污染区的温度较高, 湿度相对也较高.

空气传输轨迹的可视化分析可以了解分析轨 迹上数据的分布趋势，也可以分析不同属性之间 的相关性，进而帮助用户找出导致空气污染的最 相关因素是什么轨迹.
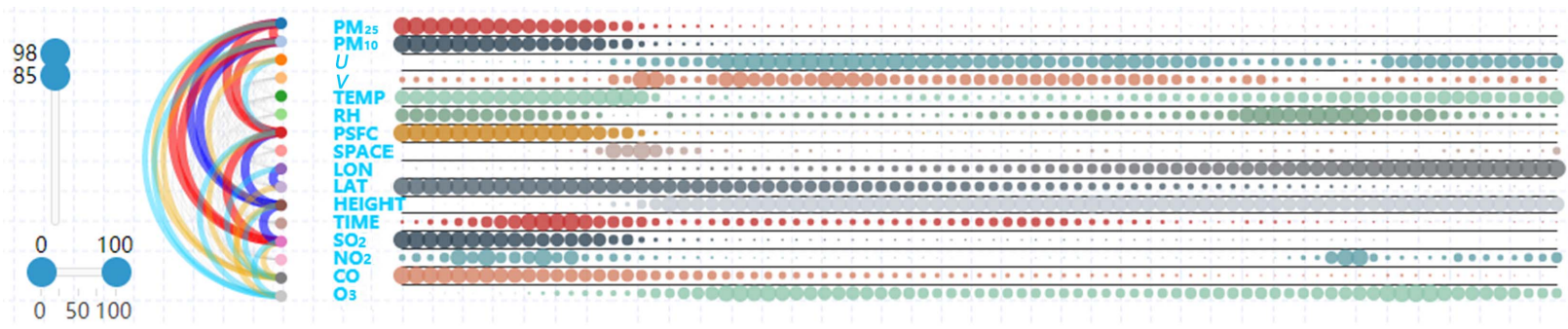

图 13 传输轨迹上的数据分布和关联 


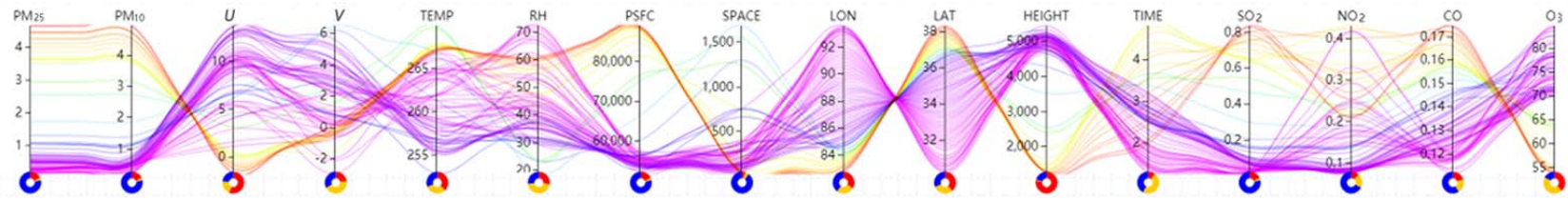

图 14 传输轨迹上的数据对比

\section{3 多因素关联分析}

多因素关联分析探索细粒度空气质量数据维 度间的内联关系. 基于变化严重程度的空气质量 多变量关联分析, 实现特定方法验证空气质量多 因素之间的相关性和相互作用特征, 如 $\mathrm{O}_{3}$ 与其他 元素的关系、污染持续增加区域的多维特征和各元 素之间的相关性分析. 这有助于研究人员快速验 证空气多因素之间的分布和相关性.

本文使用 $\mathrm{O}_{3}$, TIME 和 $\mathrm{PM}_{2.5}$ 作为主要维度, 进 行多因素关联可视化分析说明.

\subsection{1 $\mathrm{O}_{3}$ 相关分析}

$\mathrm{O}_{3}$ 是光化学污染的重要元素, 对人体有害.
从 $\mathrm{O}_{3}$ 开始相关分析, 如图 15 所示, 使用角度笔刷 工具选择一个在 $\mathrm{CO}$ 和 $\mathrm{O}_{3}$ 之间具有较大斜率的区 域, 这表明这些区域在 $\mathrm{CO}$ 和 $\mathrm{O}_{3}$ 之间具有较大反 相关性. 从图 15 中可以看出, 这些地区的污染程 度相对较轻, $\mathrm{NO}_{2}$ 和 $\mathrm{SO}_{2}$ 的含量也很低, 大部分分 布在相同的经度和纬度范围内，地形起伏不剧烈， 温度高. 同时，可以看到这些区域的风速也很小， 空气流动缓慢; 但由于 $\mathrm{SO}_{2}$ 含量低和存在其他有 害物质, $\mathrm{PM}_{2.5}$ 的含量也很低. 因此, 发现 $\mathrm{O}_{3}$ 不会 增加 $\mathrm{PM}_{2.5}$ 的含量; 同时也可以看到, 在 $\mathrm{PM}_{2.5}$ 较低 的某些地区, $\mathrm{O}_{3}$ 含量很高, 这对人体健康会造成一 定的影响.

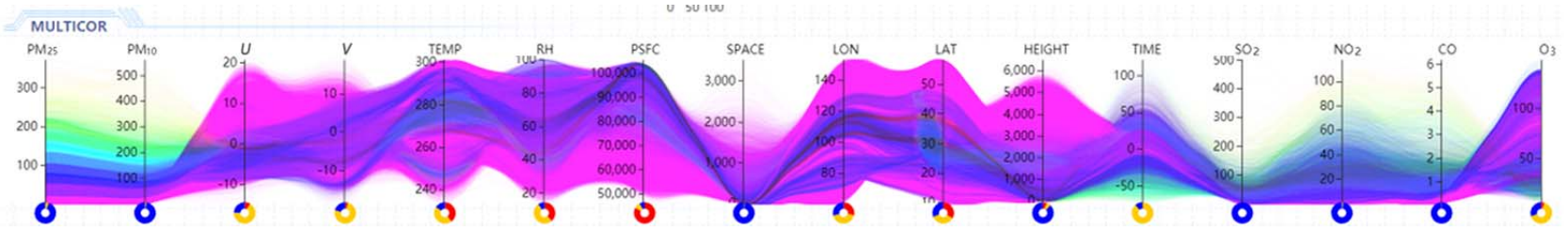

a. 初始可视化全部数据

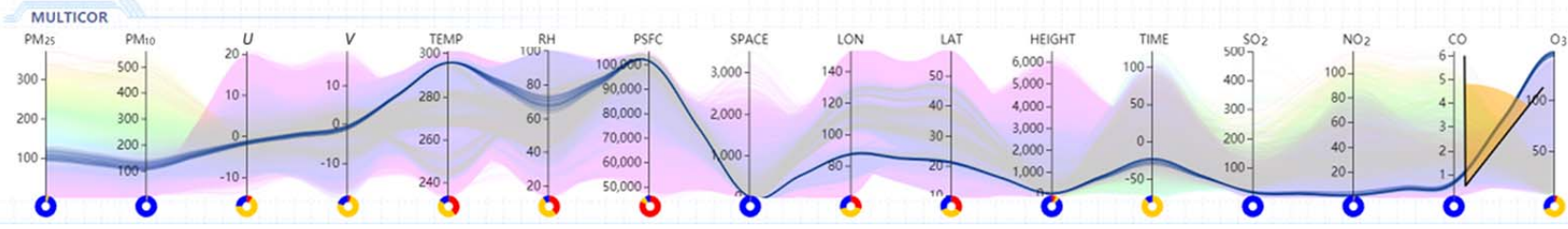

b. 使用角度笔刷选择

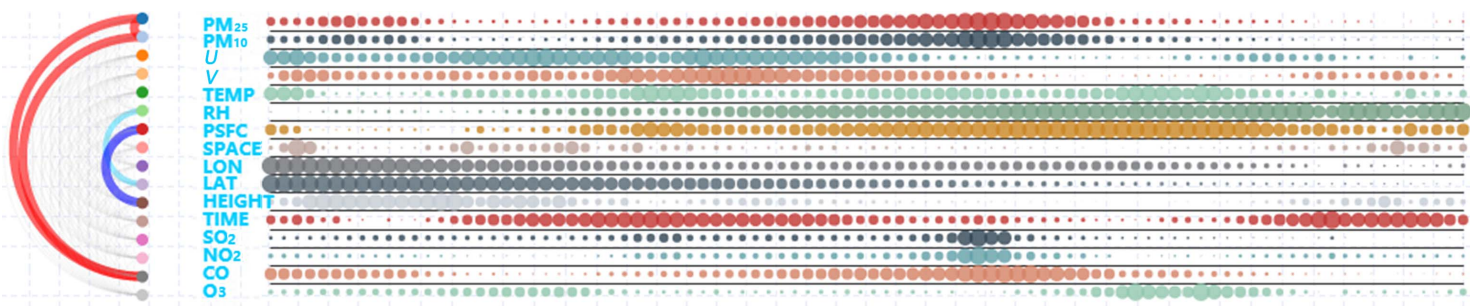

c. 角度笔刷选择后的比较视图

图 $15 \mathrm{O}_{3}$ 相关性分析

\subsection{2 污染增加的区域相关性分析}

在空气质量数据研究的过程中, 更加关注持 续污染加剧地区的分析. 如图 16 所示, 选择 1 维画 笔选择模式, 并选择 TIME 维度值较大的区域, 以 选择所有污染持续恶化的区域.

通过切换热图视图的底部图，以进行进一步的
可视化分析. 可以看出, 图 16 中圈出的区域虽然目 前的污染不是很严重, 但是有持续加剧的趋势.

\subsection{3 严重污染区的相关性分析}

从 $\mathrm{PM}_{2.5}$ 开始分析, 并使用 1 维画笔工具选择 $\mathrm{PM}_{2.5}$ 值大于 250 的区域. 如图 $17 \mathrm{a}$ 所示, 严重污染 区域中的空气流动非常缓慢, 其水平风速 $U$ 和垂 


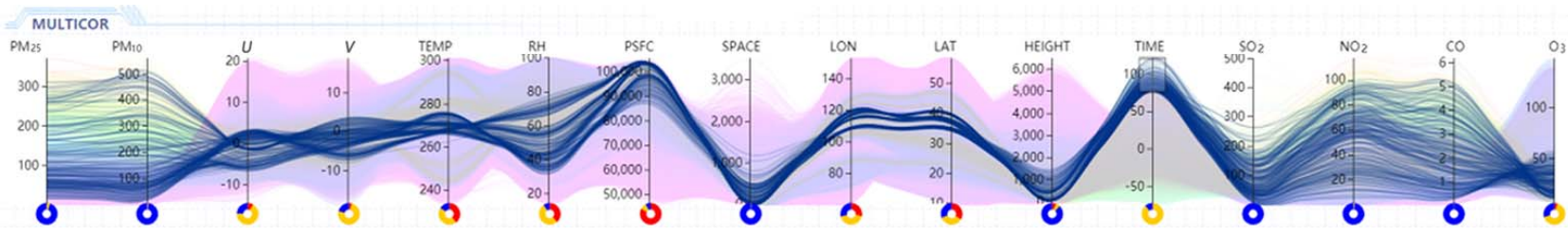

a. 选择污染持续恶化区域

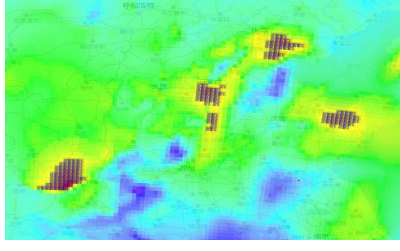

b. TIME 数据热图

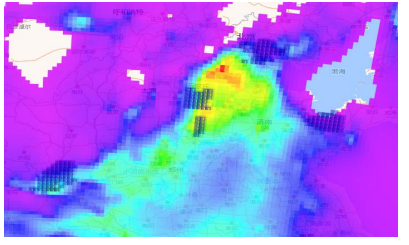

c. $\mathrm{PM}_{2.5}$ 数据热图

图 16 连续污染加剧数据的相关性分析

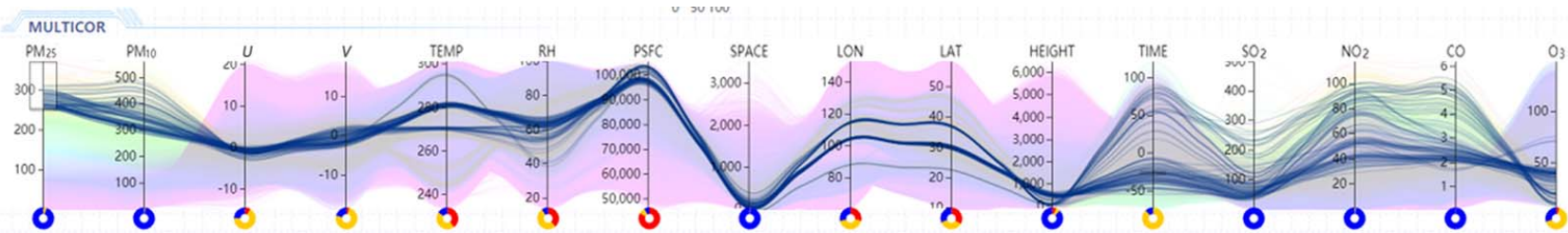

a. 选择 $\mathrm{PM}_{2.5}$ 较大区域

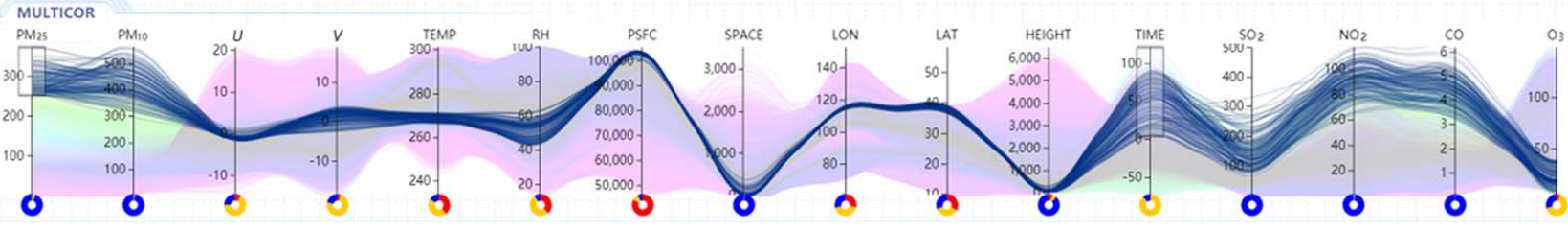

b. 选择污染持续严重区域

图 17 分析严重污染区域

直风速 $V$ 的值接近 0 ; 空间轴上的较小值表明地形 波动也相对平缓，并且 $\mathrm{SO}_{2}, \mathrm{NO}_{2}$ 和 $\mathrm{CO}$ 的含量相对 较高. 从 LON 和 LAT 轴可以看出, 数据主要分布 在 3 个区域，而气温也呈现出 3 个明显的间隔.

根据以上选择条件，可以进一步选择 TIME 轴 上较大值得到图 $17 \mathrm{~b}$, 比较将幅图可以发现, 连续 劣化的区域是 3 大污染严重地区，从热图中进一步 确定为京津冀地区.

\section{5 用户反馈}

该系统收到了来自领域科学家的积极的反馈, 用户赞赏了该系统的可用性和易用性. 系统还收 到了许多有用的建议，归类总结为 3 种代表性的反 馈：特征提取可视化效果、比较可视化效果和关联 可视化效果.

系统具有较好的特征提取可视化效果. 系统 的内联关系的建立和在此基础上的聚类方法非常 新颖, 将复杂的多维空气质量数据处理为交互式
的低维空间数据, 这有助于对高维数据进行聚类 分析, 并提供了一种技术手段, 可以深人探索数据 的深层隐藏特征. 更有趣的是, 该系统的特征数据 实际上呈现出蝴蝶形状. 这似乎是一个隐喻, 代表 着复杂的大气数据背后有蝴蝶效应.

专家经过 HAQVIS 的试用发现，该系统可以 在 Web 环境中支持大量的数据可视化和交互功能, 能够以交互方式对大量空气质量数据进行聚类分 析、比较分析和相关性分析. 特别是通过比较视图 中的比较分析功能, 可以根据自己设定的标准方 便地分析相关性, 并帮助用户快速分析污染通道 上的分布趋势. 此外, 在对于关联关系的展示上为 用户提供了更有效的分析方法，多个视图之间的 交互具有很强的可操作性. 而且, 在此系统中可以 探索许多功能, 如果将来可以添加更丰富的大气 数据, 效果会更好.

\section{6 结 语}

本文针对细粒度多维空气质量数据提出了一 
种基于深度学习的特征提取方法, 其将包括时空 特征的高维数据编码到隐含的特征维度, 并基本 保留了原始数据的特征. 然后设计了可视化分析 系统 HAQVIS，该系统集成了聚类可视化分析、关 联可视化分析、地理信息系统可视化分析，并实现 了空气污染空间划分、污染传输轨迹分析和空气质 量的细粒度内联关系可视分析．用户的反馈证明 了系统的实用性和有效性.

对于基于 VAE 的空气质量数据的可视化分析, 未来还有很多有趣的工作需要研究. 分析影响空 气质量的因素, 其数据来源主要有天气数据、人口 密度数据、生态覆被数据等. 通过将这些数据整合 到模型中，可以获得更准确的内联关系可视化分 析结果.

\section{参考文献(References):}

[1] Liu J, Han Y Q, Tang X, et al. Estimating adult mortality attributable to $\mathrm{PM}_{2.5}$ exposure in China with assimilated $\mathrm{PM}_{2.5}$ concentrations based on a ground monitoring network[J]. Science of the Total Environment, 2016, 568: 1253-1262

[2] Chai Fahe, Yun Yaru, Wang Shulan. Study on implementation of joint prevention and control of regional air pollution mechanism in China[J]. Environment and Sustainable Development, 2013, 38(4): 5-9(in Chinese)

(柴发合，云雅如，王淑兰. 关于我国落实区域大气联防联 控机制的深度思考 [J]. 环境与可持续发展, 2013, 38(4): 5-9)

[3] Wang Yonghong, Lyu Jie. Discussion on the division and control ideas of regional air pollution joint prevention and control[J]. Liaoning Urban and Rural Environmental Science \& Technology, 2015(7): 60-63(in Chinese)

(王永红，吕洁. 区域大气污染联防联控区划分及防控思路 的探讨 [J]. 环境保护与循环经济, 2015(7): 60-63)

[4] Hu Zhigao, Li Guangqin, Cao Jianhua. Air pollution regional linkage control and prevention from the perspective of environmental regulation - the scheme design, state evaluation and influencing factors analysis[J]. China Industrial Economics, 2019(5): 24-42(in Chinese)

(胡志高, 李光勤, 曹建华. 环境规制视角下的区域大气污 染联合治理一一分区方案设计、协同状态评价及影响因素分 析[J]. 中国工业经济, 2019(5): 24-42)

[5] Peng Le, Zhao Yuanyuan, Zhao Jilin, et al. Spatiotemporal patterns of air pollution transmission channel of Beijing-Tianjin-Hebei from 2000 to 2015[J]. China Environmental Science, 2019, 39(2): 449-458(in Chinese)
(彭玏, 赵媛媛, 赵吉麟, 等. 京津冀大气污染传输通道区大 气污染时空格局研究 [J]. 中国环境科学, 2019, 39(2): 449-458)

[6] Li K, Jacob D J, Liao H, et al. Anthropogenic drivers of 2013-2017 trends in summer surface ozone in China[J]. Proceedings of the National Academy of Sciences, 2019, 116(2): $422-427$

[7] Sun Yang, Feng Xiaosheng, Tang Jiuyang, et al. Survey on the research of multidimensional and multivariate data visualization[J]. Computer Science, 2008, 35(11): 1-7+59(in Chinese) (孙扬, 封孝生, 唐九阳, 等. 多维可视化技术综述 $[\mathrm{J}]$. 计算 机科学, 2008, 35(11): 1-7+59)

[8] Qu H M, Chan W Y, Xu A B, et al. Visual analysis of the air pollution problem in Hong Kong[J]. IEEE Transactions on Visualization and Computer Graphics, 2007, 13(6): 1408-1415

[9] Treinish L A. Multi-resolution visualization techniques for nested weather models $[\mathrm{C}] / /$ Proceedings of the IEEE Visualization. Los Alamitos: IEEE Computer Society Press, 2000: 513-516

[10] Völgyesi P, Nádas A, Koutsoukos X, et al. Air quality monitoring with SensorMap[C] //Proceedings of International Conference on Information Processing in Sensor Networks. Los Alamitos: IEEE Computer Society Press, 2008: 529-530

[11] Treinish L A. Visual data fusion for applications of high-resolution numerical weather prediction[C] //Proceedings of the IEEE Visualization. Los Alamitos: IEEE Computer Society Press, 2000: 477-480

[12] Healey C G, Tateosian L, Enns J T, et al. Perceptually based brush strokes for nonphotorealistic visualization[J]. ACM Transactions on Graphics, 2004, 23(1): 64-96

[13] Ito K, Thurston G D, Nadas A, et al. Monitor-to-monitor temporal correlation of air pollution and weather variables in the North-Central U.S.[J]. Journal of Exposure Science \& Environmental Epidemiology, 2001, 11: 21-32

[14] Tang Y, Qu H M, Wu Y C, et al. Natural textures for weather data visualization[C] //Proceedings of the International Conference on Information Visualization. Los Alamitos: IEEE Computer Society Press, 2006: 741-750

[15] Demuzere M, Trigo R M, de Arellano J V G, et al. The impact of weather and atmospheric circulation on $\mathrm{O}_{3}$ and $\mathrm{PM}_{10}$ levels at a rural mid-latitude site[J]. Atmospheric Chemistry and Physics, 2009, 9(167): 2695-2714

[16] Quinan P S, Meyer M. Visually comparing weather features in forecasts[J]. IEEE Transactions on Visualization and Computer Graphics, 2016, 22(1): 389-398

[17] Tang X, Kong L, Zhu J, et al. A six-year long high-resolution air quality reanalysis dataset over China from 2013 to 2018 . V1[OL]. [2021-03-08]. http://www.doi.org/10.11922/sciencedb.00053

[18] Kingma D P, Welling M. Auto-encoding variational Bayes[OL]. [2021-03-08]. https://arxiv.org/pdf/1312.6114v10.pdf 